\title{
CONCERNING RINGS OF CONTINUOUS FUNCTIONS
}

\author{
BY \\ LEONARD GILLMAN AND MELVIN HENRIKSEN
}

The present paper deals with two distinct, though related, questions, concerning the ring $C(X, R)$ of all continuous real-valued functions on a completely regular topological space $X$.

The first of these, treated in $\$ \S 1-7$, is the study of what we call $P$-spacesthose spaces $X$ such that every prime ideal of the ring $C(X, R)$ is a maximal ideal. The background and motivation for this problem are set forth in $\$ 1$. The results consist of a number of theorems concerning prime ideals of the ring $C(X, R)$ in general, as well as a series of characterizations of $P$-spaces in particular.

The second problem, discussed in $\$ \S 8-10$, is an investigation of what Hewitt has termed $Q$-spaces-those spaces $X$ that cannot be imbedded as a dense subset of any larger space over which every function in $C(X, R)$ can be continuously extended. An introduction to this question is furnished in $\S 8$. Our discussion of $Q$-spaces is confined to the class of linearly ordered spaces (introduced in $\$ 6$ ). We are able to settle the question as to when an arbitrary linearly ordered space is or is not a $Q$-space. The concept of a paracompact space turns out to be intimately related to these considerations. We also derive a characterization of linearly ordered paracompact spaces, and we find in particular that every linearly ordered $Q$-space is paracompact. A result obtained along the way is that every linearly ordered space is countably paracompact.

1. Introduction ( $P$-spaces). Kaplansky has pointed out [12, Theorem 30$]$ that if $X$ is discrete, then every prime ideal of the ring $C=C(X, R)$ is maximal. Here we examine what topological consequences this property of the ring $C$ has for the space $X$. We find that if every prime ideal of $C$ is maximal (or merely every prime fixed ideal-definition below) then $X$ has the following feature in common with discrete spaces: every continuous real-valued function vanishing at a point $p$ vanishes on a neighborhood of $p$.

The converse is also valid. Such spaces, then, might be termed "pseudodiscrete." We call them, for brevity, "P-spaces." These constitute a much wider class than the discrete spaces: examples abound of $P$-spaces that have no isolated points whatsoever. The distinction is easily expressed in algebraic terms: a $P$-space $X$ is discrete if and only if every maximal fixed ideal of $C(X, R)$ is principal (Theorem 5.9).

Among the additional characterizations of general $P$-spaces $X$ that we

Presented to the Society, April 25, 1953; received by the editors June 9, 1953 and, in revised form, July 21, 1953. 
obtain are the following (Theorem 5.3): every countable intersection of open sets is open; $C(X, R)$ is a regular ring; every ideal of $C(X, R)$ is an intersection of maximal ideals; every ideal of $C(X, R)$ is closed in the $m$-topology of Hewitt (for definition see $\$ 5$ ).

2. Terminology and notation. Familiarity with the contents of [10] will be helpful, but an effort has been made to keep the paper reasonably selfcontained. All topological spaces $X$ considered will be assumed to be completely regular and Hausdorff( $\left.{ }^{1}\right)$. Let $f$ be any function in the ring $C(X, R)$; the set $Z(f)$ of all zeros of $f$ is called the zero-set of $f$. It follows from complete regularity that every open set of $X$ contains a zero-set. It is easily seen that every zero-set is a $G_{\delta}$ (i.e., a countable intersection of open sets). Conversely, if $X$ is normal then every closed $G_{\delta}$ is a zero-set; but there are non-normal completely regular spaces in which not every closed $G_{\delta}$ is a zero-set $[10$, p. 69]. For any subset $A$ of $C$, we put $Z(A)=\{Z(f) \mid f \in A\}$. The subring of all bounded functions of the ring $C=C(X, R)$ is denoted by $C^{*}=C^{*}(X, R)$.

An ideal $I$ of $C$ (or $C^{*}$ ) is called fixed if the set $\bigcap_{f \in I} Z(f)$ is nonempty. Otherwise, $I$ is called a free ideal. If $p \in \bigcap_{f \in I} Z(f)$, we shall call $I$ an ideal at $p$. It is well known that the only maximal ideal of $C$ at a point $p$ is the set

$$
M_{p}=\{f \mid f \in C, f(p)=0\} .
$$

The ring $C$ (or $C^{*}$ ) contains a free ideal if and only if $X$ is not compact( ${ }^{2}$ ) $[10$, Theorems 7, 37].

Let $I$ be any proper ideal of $C$, and $\mathcal{Z}=\mathcal{Z}(I)$; then (1) $\mathcal{Z}$ has the finite intersection property, i.e., it is closed under finite intersection and it does not contain the empty set, and (2) $Z$ is closed under extension, i.e., if $Z \in Z, Z^{\prime} \in Z(C)$, $Z^{\prime} \supset Z$, then $Z^{\prime} \in Z$. Conversely, if (1) and (2) hold for any subfamily $Z$ of $Z(C)$, then $Z=Z(I)$ for some ideal $I$ of $C$. The ideal $I$ is maximal if and only if, in addition to (1) and (2), we have (3) if $Z^{\prime} \in Z$ there is a $Z \in Z$ such that $Z \cap Z^{\prime}$ is empty [10, Theorem 36]. Therefore if $Z\left(f_{1}\right) \cup Z\left(f_{2}\right) \supset Z(f)$, where $f$ belongs to a maximal ideal $M$, then either $f_{1} \in M$ or $f_{2} \in M$ (since $Z\left(f_{1} f_{2}\right)$ $=Z\left(f_{1}\right) \cup Z\left(f_{2}\right)$, and $M$ is prime).

It is well known $[2 ; 21]$ that every completely regular space $X$ can be imbedded as an everywhere dense subset of a compact space $\beta X$ (the Stone-Čech compactification of $X)$ such that every $f \in C^{*}(X, R)$ has a unique continuous extension to $C^{*}(\beta X, R)=C(\beta X, R)$. This extension of $f$ will be denoted by $\bar{f}$. For any subset $S$ of $X$, the closure of $S$ in $\beta X$ will be denoted by $\bar{S}$. In particular, the closure in $\beta X$ of a zero-set $Z(f)$ (which is a closed subset of $X)$ is denoted by $\bar{Z}(f)$. Note that $\bar{Z}(f) \subset Z(\bar{f})$ (if $\bar{f}$ exists).

If $\Omega$ is any open subset of $\beta X$, then for any $p \in \Omega$, we shall call $\Omega \cap X$ an $X$-neighborhood of $p$.

(1) For general references in topology see [15, Chap. I]; all topological spaces considered in the present paper will be assumed to consist of more than one point. For references in algebra see $[26$, Volume $I ; 16]$.

(2) Hewitt, in [10], uses the term bicompact for our compact. 
Since $C^{*}(X, R)$ and $C(\beta X, R)$ are isomorphic, every maximal ideal $M^{*}$ of $C^{*}(X, R)$ assumes the form $M^{*}=M^{* p}=\left\{f \mid f \in C^{*}(X, R), \bar{f}(p)=0\right\}$ for some $p \in \beta X$; and $M^{* p}$ is fixed or free according as $p \in X$ or $p \in \beta X-X$.

Definition 2.1. For every point $p$ of $\beta X$, we denote by $N^{p}$ the ideal of $C(X, R)$ consisting of all $f \in C(X, R)$ such that $Z(f)$ contains an $X$-neighborhood of $p$.

When $p \in X$, and when this fact deserves emphasis, we write $N_{p}$ in place of $N^{p}$. Note that if $p \in X$, then $N_{p}$ consists of all $f \in C(X, R)$ that vanish on a neighborhood (in $X$ ) of $p$. On the other hand, if $p \in \beta X-X$, then $N^{p}$ is easily seen to be a free ideal.

From now on, unless otherwise stated, all ideals referred to are ideals of $C(X, R)$.

For any cardinal number $\mathfrak{m}$, we write exp $\mathfrak{m}$ to denote the cardinal $2^{\mathfrak{m}}$.

3. Prime ideals of the ring $C(X, R)$. The following lemma, due to Gelfand and Kolmogoroff, is essential for the development below. A proof of the lemma is given in [5].

Lemma 3.1 (Gelfand-Kolmogoroff). For every point $p$ of $\beta X$, the set

$$
M^{p}=\{f \mid f \in C(X, R), p \in \bar{Z}(f)\}
$$

is a maximal ideal of $C(X, R)$. Conversely, for every maximal ideal $M$ of $C(X, R)$ there is a unique $p \in \beta X$ such that $M=M^{p}$. If $p \in X$, then $M^{p}$ is the fixed ideal $M_{p}$; otherwise $M^{p}$ is free.

Lemma 3.2. If $P$ is any prime ideal contained in the maximal ideal $M^{p}$, then $P \supset N^{p}$.

Proof. Let $f \in N^{p}$. By definition of $N^{p}$, there is an open subset $\Omega$ of $\beta X$ such that $p \in U=\Omega \cap X$, and $f(U)=0$. Since $\beta X$ is completely regular, there is a $g \in C^{*}(X, R)$ such that $\bar{g}(p)=1, \bar{g}(\beta X-\Omega)=0$. Clearly $f g=0$. Since $P$ is prime and $g \notin M^{p}$, we must have $f \in P$. It follows that $N^{p} \subset P$.

TheOREM 3.3. For any completely regular space $X$, every prime ideal $P$ of $C(X, R)\left(P^{*}\right.$ of $\left.C^{*}(X, R)\right)$ is contained in a unique maximal ideal of $C(X, R)$ $\left(C^{*}(X, R)\right)$.

Proof. Since $C$ is a ring with unit, the prime ideal $P$ is contained in at least one maximal ideal, say $M^{p}$. Let $q$ be any point of $\beta X$ distinct from $p$; it suffices to show that $P$ is not contained in the maximal ideal $M^{q}$. Let $\Omega_{p}, \Omega_{q}$ be disjoint neighborhoods (in $\beta X$ ) of $p, q$, respectively. By complete regularity, there is an $f \in C^{*}(X, R)$ such that $\bar{f}(q)=1, \bar{f}\left(\beta X-\Omega_{q}\right)=0$. Since $\beta X-\Omega_{q} \supset \Omega_{p}$, $f$ is in $N^{p}$ and clearly is not in $M^{q}$. But by Lemma 3.2, $N^{p} \subset P$. Hence $P$ is not contained in $M^{q}$. This establishes the result for $C(X, R)$. Applied to $C(\beta X, R)$, it yields the theorem for $C^{*}(X, R)$.

Note that we have shown that $N^{p}$ is contained in a unique maximal ideal, namely $M^{p}$. 
COROllary 3.4. The residue class ring $C / P$ of $C$ with respect to any prime ideal $P$ contains exactly one maximal ideal (namely, $M^{p} / P$ ); this ideal consists of all non-units of $C / P$. The corresponding statement holds for $C^{*}$.

Since the square root of a non-negative continuous function is continuous, it may be seen without difficulty that $M^{2}=M$ for every maximal ideal $M$ of $C(X, R)$. This is fundamentally different from the situation in the ring of entire functions-see [8].

THEOREM 3.5. For every point $p$ of $\beta X$, the following two statements are equivalent.

(1) $M^{p}$ is the only prime ideal containing $N^{p}$.

(2) $M^{p}=N^{p}$ (i.e., every continuous function $f$ such that $p \in \bar{Z}(f)$ vanishes on an $X$-neighborhood of $p$ ).

Proof. That (2) implies (1) follows directly from Lemma 3.2. For the converse, suppose that $f \in M^{p}-N^{p}$. Since $Z\left(f^{n}\right)=Z(f)$ for every positive integer $n$, we have $f^{n} \in M^{p}-N^{p}$ for all such $n$. Hence the set $S$ of positive powers of $f$ forms a multiplicative system that does not meet $N^{p}$. Consequently there is a prime ideal $P$ containing $N^{p}$ that does not meet $S$ (see, e.g., $[16$, p. 105]). Obviously $P \neq M^{p}$.

We show next that if there is an unbounded $f$ in $C(X, R)$, then the ring $C^{*}(X, R)$ always has at least one nonmaximal prime ideal. In fact, the referee has pointed out the following stronger result.

Theorem 3.6. If $C(X, R) \neq C^{*}(X, R)$, then $C^{*}(X, R)$ has at least $\exp \exp \aleph_{0}$ nonmaximal prime free ideals.

Proof. If $f$ is any unbounded function of $C(X, R)$ then $g=e^{-f^{2}}$ is in $C^{*}(X, R)$ and vanishes nowhere on $X$, but assumes arbitrarily small values. Consequently, $Z(\bar{g})$ is nonempty and is contained in $\beta X-X$. But $X$ is everywhere dense in $\beta X$, whence $Z(\bar{g})$ has a void interior. By Theorem 3.5 , there is a nonmaximal prime ideal $P^{p}$ of $C(\beta X, R)$ at any point $p \in Z(\bar{g})$. Since $C^{*}(X, R)$ is isomorphic with $C(\beta X, R)$, the corresponding prime ideal $P$ of $C^{*}(X, R)$ is nonmaximal. Since $p \in \beta X-X$, the ideal $P$ is free. Now Theorem 3.3 shows that $P$ is contained in the unique maximal ideal $M^{* p}$ of $C^{*}$; and by [10, Theorem 49], the cardinal of $Z(\bar{g})$ is at least exp exp $\aleph_{0}$. It follows that $C^{*}$ contains at least $\exp \exp \aleph_{0}$ nonmaximal prime free ideals.

A related result is stated in Theorem 5.10 below.

REMARK 3.7. More explicit examples of nonmaximal prime ideals of $C^{*}$ are given in [5, Theorem $6 \mathrm{ff}$.]; in particular, it can be inferred that for a discrete $Q$-space $X$ of power $\aleph_{\alpha}$ (see $\S 8$ below), $C^{*}(X, R)$ has $\exp \exp \aleph_{\alpha}$ nonmaximal prime ideals.

\section{P-points.}

Definition 4.1. A point $p \in X$ is called a $P$-point if the only prime ideal of $C(X, R)$ at $p$ is the maximal ideal $M_{p}$. 
THEOREM 4.2. For every point $p \in X$, the following statements are equivalent.

(1) $p$ is a P-point.

(2) Every continuous function vanishing at $p$ vanishes on a neighborhood of p, i.e., $M_{p}=N_{p}$.

(3) Every countable intersection of neighborhoods of $p$ contains a neighborhood of $p$.

Proof. The equivalence of (1) and (2) is simply a special case of Theorem 3.5. Suppose (3) holds. Since

$$
Z(f)=\bigcap_{n=1}^{\infty}\left\{x|| f(x) \mid<\frac{1}{n}\right\},
$$

$f(p)=0$ implies that $f$ vanishes on a neighborhood of $p$. So (3) implies (2). For the converse, we first recall that the intersection of countably many zerosets is a zero-set [10, Theorem 33]. (If $\left\{f_{n}\right\}$ is a sequence of elements of $C$, then $g_{n}=\min \left\{\left|f_{n}\right|, 1\right\}$ is continuous and $0 \leqq g_{n} \leqq 1$, and $Z\left(g_{n}\right)=Z\left(f_{n}\right)$. Then $g=\sum_{n=1}^{\infty} 2^{-n} g_{n}$ is continuous and $Z(g)=\bigcap_{n=1}^{\infty} Z\left(f_{n}\right)$.) Now let $p$ be any $P$ point and $\left\{U_{n}\right\}$ any sequence of neighborhoods of $p$. By complete regularity, every $U_{n}$ contains a zero-set $Z_{n}$ containing $p$. Thus $\bigcap_{n=1}^{\infty} U_{n} \supset \cap_{n=1}^{\infty} Z_{n}=Z(f)$ for some $f \in C$, and $f(p)=0$. By (2), $Z(f)$ contains a neighborhood of $p$; hence (3) holds.

This theorem can be generalized to refer to any point of $\beta X$. In giving such a formulation, one would first define a point $p \in \beta X$ to be a $P$-point with respect to $X$ if every $f \in M^{p}$ vanishes on an $X$-neighborhood of $p$.

Corollary 4.3. Every isolated point is a P-point. Conversely, if the P-point $p$ is a $G_{\delta}$ (in particular, if the first axiom of countability holds at $p$ ), then $p$ is an isolated point.

THEOREM 4.4. If the non-isolated point $p \in X$ has a base of neighborhoods linearly ordered by set-inclusion, then $p$ is a P-point if and only if the first axiom of countability fails at $p$.

Proof. Let $U=\left\{U_{\sigma}\right\}$ be a base of neighborhoods at $p$ that is linearly ordered by set-inclusion. If the first axiom of countability holds at $p$, then by the corollary above, $p$ cannot be a $P$-point unless it is isolated. Conversely, let $\left\{V_{n}\right\}$ be any countable family of neighborhoods of $p$. For each $n$ there is a $U_{\sigma_{n}} \in U$ such that $V_{n} \supset U_{\sigma_{n}}$. Then $\bigcap_{n=1}^{\infty} V_{n} \supset \cap_{n=1}^{\infty} U_{\sigma_{n}}$, and the latter contains some neighborhood $U_{\sigma} \in U$. By Theorem 4.2, $p$ is a $P$-point.

Example 7.5 below shows that a $P$-point need not have a linearly ordered base of neighborhoods.

5. $P$-spaces.

Definition 5.1. $X$ is called a $P$-space if every point of $X$ is a $P$-point, i.e., every prime fixed ideal of $C(X, R)$ is maximal.

Two of our characterizations of $P$-spaces will be in terms of what Hewitt 
has called the $m$-topology $[10$, p. 49$]$ on the ring $C(X, R)$. Here a neighborhood for a base at $f \in C$ is defined to be any set of the following form: all $g \in C$ such that $|f(x)-g(x)|<\pi(x)$ for all $x \in X$, where $\pi$ is any function in $C$ that is everywhere positive. Hewitt has shown that every maximal ideal of $C$ is $m$-closed [ 10 , Theorem 38], and has conjectured that every $m$-closed ideal is an intersection of maximal ideals. This conjecture has been confirmed by the present writers, jointly with M. Jerison [5, Theorem 9]:

THEOREM 5.2. Every $m$-closed ideal of $C$ is the intersection of all the maximal ideals containing it.

The main result of this section is the following.

THEOREM 5.3. The following statements are equivalent.

(1) $X$ is a $P$-space.

(2) Every zero-set of $X$ is open (and closed).

(3) $C(X, R)$ is a regular ring $\left({ }^{3}\right)$.

(4) Every prime ideal (free or fixed) of $C(X, R)$ is maximal.

(5) Every countable intersection of open sets of $X$ is open (every countable union of closed sets is closed).

(6) Every ideal of $C(X, R)$ is the intersection of all the maximal ideals containing it.

(7) Every ideal of $C(X, R)$ is m-closed.

(8) Every prime fixed ideal of $C(X, R)$ is m-closed.

Proof. (1) implies (2). By Theorem 4.2, for every $f$ in $C(X, R), f$ vanishes on a neighborhood of every point of $Z(f)$. Hence $Z(f)$ is open.

(2) implies (3). Let $f$ be any element of $C$. Define $g$ as follows: $g=0$ on $Z(f), g=1 / f$ on $X-Z(f)$. Since $Z(f)$ is open and closed, $g$ is continuous. Clearly $f^{2} g=f$. It follows that the ring $C$ is regular.

(3) implies (4). Obviously every homomorphic image of a regular ring is regular, and clearly any regular integral domain is a field. It follows that every prime ideal of a commutative regular ring with unit is maximal. (One may also show directly that (2) implies (4) by using the argument given by Kaplansky in [12, Theorem 30].)

That (4) implies (1) is obvious from the definition of a $P$-space. This establishes the equivalence of (1), (2), (3), and (4).

(1) implies (5). Let $\left\{U_{n}\right\}$ be any countable family of open sets, and put $U=\bigcap_{n=1}^{\infty} U_{n}$. By Theorem 4.2, there is for any $x \in U$ a neighborhood of $x$ contained in $U$. Hence $U$ is open.

(5) implies (1) by Theorem 4.2. There remain the equivalences with (6), $(7)$, and (8).

(3) implies (6). As noted in [1, p. 459], every ideal of a commutative regular ring is the intersection of all the maximal ideals containing it.

(3) A ring $A$ is called regular if for every $a \in A$ there is an $x \in A$ such that $a x a=a$. 

38 ].

(6) implies (7). Every maximal ideal of $C(X, R)$ is $m$-closed $[10$, Theorem

That (7) implies (8) is trivial.

(8) implies (1). Suppose that $X$ is not a $P$-space. Then by Theorem 4.2, there is a $p \in X$ and a prime ideal $P$ at $p$ such that $P \subset M_{p}$ and $P \neq M_{p}$. Now $M_{p}$, being maximal, is $m$-closed, and from Theorem 3.3 it is the only maximal ideal containing $P$. Theorem 5.2 now implies that $P$ is not $m$-closed. This completes the proof.

An additional characterization of a $P$-space $X$ is that every point of $\beta X$ be a $P$-point with respect to $X$ (cf. the remark following Theorem 4.2).

Since every neighborhood of a point contains a zero-set containing the point, it is plain that condition (2) of the theorem may be phrased as follows: the family of zero-sets constitutes a base of open sets for the space.

Spaces with the property (5) have been called $\omega_{1}$-additive by Sikorski [19]; he points out that in such spaces every countable set is closed and discrete. Hence no infinite subset of a $P$-space can be countably compact. So we have:

CoRollary 5.4. Every countably compact P-space is finite; every locally countably compact $P$-space is discrete.

It can happen that $C^{*}(X, R)=C(X, R)$ and yet $X$ is not countably compact [10, Theorem 29]. But then $X$ cannot be a $P$-space:

Corollary 5.5. If $X$ is a $P$-space and $C(X, R)=C^{*}(X, R)$, then $X$ is finite.

Proof. $X \subset \beta X$, and the hypotheses imply that the compact space $\beta X$ is a $P$-space.

Corollary 5.6. Every subspace of a $P$-space is a $P$-space.

Corollary 5.7. An arbitrary sum( $\left(^{4}\right)$ of $P$-spaces is a $P$-space.

Corollary 5.8. Every finite product of $P$-spaces is a $P$-space, but an infinite product need not be.

Proof. The first statement is an immediate consequence of (5) of the theorem. The second part follows from the observation that by the Tychonoff theorem [15] the product of infinitely many finite (hence compact) $P$-spaces is an infinite compact space.

We can easily give an example of a space $X$ for which every prime free ideal is maximal and yet $X$ is not a $P$-space. Let $X$ be the sum of an infinite compact space $X_{1}$ and an infinite $P$-space $X_{2}$. Then clearly $C(X, R)$ is the direct sum of $C\left(X_{1}, R\right)$ with $C\left(X_{2}, R\right)$, so that $C(X, R)$ has nonmaximal prime ideals (those contributed by $C\left(X_{1}, R\right)$ ), but every prime free ideal is maximal,

(4) A topological space $X$ is said to be the sum of the spaces $X_{\alpha}$ provided the $X_{\alpha}$ are mutually disjoint, $X=\cup_{\alpha} X_{\alpha}$, and each $X_{\alpha}$ is open and closed in $X$. 
since $C\left(X_{1}, R\right)$ has none ( $X_{1}$ being compact), and $X_{2}$ is a $P$-space.

Theorem 5.9. A $P$-space $X$ is discrete if and only if every maximal fixed ideal of $C(X, R)$ is principal.

Proof. Suppose that $X$ is discrete, and let $M_{p}$ be any maximal fixed ideal of $C$. Define $g \in C$ by: $g(p)=0, g(q)=1$ if $q \neq p$. Then $f=f g$ for every $f \in M_{p}$. Hence $M_{p}$ is the principal ideal $(g)$.

Conversely, let $p$ be any point of $X$. By hypothesis, $M_{p}=(g)$ for some $g$. Hence for every $f \in M_{p}$, we have $Z(f) \supset Z(g)$. Therefore $\cap_{f \in M_{p}} Z(f) \supset Z(g)$. But $\cap_{f \in M_{p}} Z(f)=\{p\}$. So $\{p\} \supset Z(g)$. But $Z(g)$ is open, since $X$ is a $P$-space; and $Z(g)$ is nonempty, since the ideal $(g)$ is proper. Consequently $\{p\}$ is an open set. Thus $X$ is discrete.

As a direct consequence of Theorem 3.6 and Corollary 5.5, we obtain the following result for arbitrary completely regular spaces.

THEOREM 5.10. Let $X$ be any completely regular space; then $C^{*}(X, R)$ contains a nonmaximal prime ideal if and only if $X$ is infinite.

6. Linearly ordered spaces. By a linearly ordered space we mean any linearly ordered set provided with its interval topology $\left(^{(5)}\right.$. It is well known that every such space is normal. The remainder of our paper is concerned chiefly with these spaces.

We first recall some concepts from the theory of linearly ordered sets, for which reference may be made to Hausdorff [7]. The set of all ordinals less than a given ordinal $\phi$ is denoted by $W(\phi)$. The initial ordinal of the cardinal $\boldsymbol{\aleph}_{\alpha}$ is denoted by $\omega_{\alpha}\left(\omega_{0}=\omega\right)$. If $W\left(\omega_{\alpha}\right)$ is cofinal with no subset of type $<\omega_{\alpha}$, then $\omega_{\alpha}$ and $\boldsymbol{\aleph}_{\alpha}$ are said to be regular; otherwise they are singular. The number $\omega$ is regular, and so is every initial ordinal of the form $\omega_{\beta+1}$.

Let $L$ be any linearly ordered set. A gap of $L$ is customarily defined as a Dedekind cut $(A \mid B)$ of $L$ such that $A$ has no last element and $B$ no first; it may be regarded as a "virtual" element $u$ that satisfies the ordering relations $a<u<b$ for all $a \in A$ and $b \in B$. We shall call this an interior gap. If the set $L$ itself has no first (last) element, we shall introduce a virtual element $u$ such that $u<x(u>x)$ for all $x \in L$, and refer to $u$ as a left (right) end-gap. The linearly ordered set consisting of all elements and all gaps (end-gaps as well as interior gaps) of $L$ will be denoted by $L^{+}$.

For every nonvoid subset $S$ of $L^{+}$there exists a least element $k$ of $L^{+}$ such that $k \geqq s$ for all $s \in S$, and a greatest element $h$ of $L^{+}$such that $h \leqq s$ for all $s \in S$; we write, as usual, $k=\sup S, h=\inf S$. In case $S$ is an increasing (or decreasing) sequence having no last term, we also call $k$ (or $h$ ) the limit of $S$,

(5) Observe that a subspace of a linearly ordered space need not be a linearly ordered space. For example, let $X$ be obtained from the reals by deleting the interval $(0,1]$. In the interval topology of $X$, every neighborhood of 0 contains points $>1$, but this is not true in the relative topology on $X$. 
and write $k($ or $h)=\lim S$.

Let $L$ be a linearly ordered set of more than one element, and let $u$ be any element of $L^{+} ; u$ is called an $\omega_{\alpha^{-}}\left(\omega_{\beta^{-}}^{*}\right)$ limit of $L$ if $\omega_{\alpha}\left(\omega_{\beta}\right)$ is regular, and if the set of all elements of $L$ that precede (follow) $u$ is cofinal (coinitial) with $\omega_{\alpha}\left(\omega_{\beta}^{*}\right)\left(^{*}\right.$ signifies inverse order). If $u$ is a limit from the left (right), then it is an $\omega_{\alpha^{-}}\left(\omega_{\beta^{-}}^{*}\right)$ limit for unique $\alpha(\beta)$. Associated with every $u \in L^{+}$is an ordered pair $\left\langle\rho, \sigma^{*}\right\rangle$, called its character, which we define as follows: (1) if $u$ is an $\omega_{\alpha^{-}}\left(\omega_{\beta^{-}}^{*}\right)$ limit, then $\rho=\omega_{\alpha}\left(\sigma=\omega_{\beta}\right)$; (2) if $u$ has an immediate predecessor (successor), then $\rho=1(\sigma=1)$; (3) if $u$ is the first (last) element of $L^{+}$, then $\sigma(\rho)$ has already been defined in (1) or (2), and we put $\rho=\sigma(\sigma=\rho)$. A character of the form $\left\langle\omega_{\alpha}, \omega_{\beta}^{*}\right\rangle$ is also denoted by $c_{\alpha \beta}$; in case $\alpha=\beta$, the corresponding element or gap is called symmetric.

The symbols ( ), [ ], etc., will be employed in the usual way to denote intervals of $L$ (open, closed, etc.). The indicated boundaries of the interval will be in $L^{+}$, but they need not be in $L$ itself.

The linearly ordered set $L$ is dense if between any two of its elements there lies another.

We shall make use of a fundamental existence theorem due to Hausdorff [6, Theorems XVI and XVII], which may be stated as follows.

THEOREM 6.1. (HAUSDORFF). Let $\sigma, \tau$ be any ordinals. Let $T$ be any subset of the Cartesian product $W(\sigma+1) \otimes W(\tau+1)$ such that the ordered pair $\langle\alpha, \beta\rangle$ is in $T$ only if both $\omega_{\alpha}$ and $\omega_{\beta}$ are regular, and, subject to this restriction, such that

(1) for every $\alpha \leqq \sigma$ there is a $\beta \leqq \tau$ such that $\langle\alpha, \beta\rangle \in T$,

(2) for every $\beta \leqq \tau$ there is an $\alpha \leqq \sigma$ such that $\langle\alpha, \beta\rangle \in T$,

(3) there is an $\langle\alpha, \beta\rangle \in T$ for which $\alpha=\beta$.

Let $K$ denote the set of all characters $c_{\alpha \beta}=\left\langle\omega_{\alpha}, \omega_{\beta}^{*}\right\rangle$ for which $\langle\alpha, \beta\rangle \in T$. Finally, let $E, G$ be any subsets of $K$, whose union is $K$, and with $E$ nonempty.

Then there exists a (dense) linearly ordered set $L$, such that for every open interval $J$ of $L$, the set of element characters of $J$ is precisely the set $E$, and the set of gap characters of $J$ is precisely $G$.

We close this section with a lemma which will be needed subsequently. The statement of this lemma is in terms of increasing sequences; here and in similar instances we omit the obvious remark that the corresponding result for decreasing sequences is also valid.

Lemma 6.2. Let $X$ be any linearly ordered space. Denote its first element or gap by $a$, and its last by $b$, and let $u$ be an arbitrary element or gap of $X$. If $u>a$ and $u$ has no immediate predecessor, then there is an increasing sequence $T$ $=\left\{x_{\xi}\right\}$, with limit $u$, such that every closed interval $\left[x_{\xi}, b\right]$ is a zero-set of $X$. (If in addition $u$ is a gap, then the intervals $\left[x_{\xi}, u\right]$ are also zero-sets.)

Proof. The parenthetical remark follows from the fact that if $u$ is a gap, then the interval $[a, u]$ is open and closed, hence a zero-set, whence so is each 
intersection $\left[x_{\xi}, u\right]=[a, u] \cap\left[x_{\xi}, b\right]$. Now in a normal space every closed $G_{\delta}$ is a zero-set $(\S 2)$. Hence $[x, b]$ is a zero-set for every $\omega$-limit $x$, as well as for every $x$ that has an immediate predecessor. Let $\alpha$ denote the ordinal for which $u$ is an $\omega_{\alpha}$-limit, and let $S=\left\{y_{\xi}\right\}_{\xi<\omega_{\alpha}}$ be any increasing sequence of type $\omega_{\alpha}$ whose limit is $u$. If $\alpha>0$, the required sequence $T$ may be taken as the set of $\omega$-limits of the set $S$. For $\alpha=0$, we define $x_{n}(n<\omega)$ as follows. If $y_{n+1}$ has an immediate predecessor, we take the latter as $x_{n}$. If $y_{n+1}$ is an $\omega$-limit, we put $x_{n}=y_{n+1}$. Finally, if $y_{n+1}$ is an $\omega_{\alpha}$-limit for some $\alpha>0$, then we take as $x_{n}$ any point satisfying $y_{n}<x_{n}<y_{n+1}$ for which $\left[x_{n}, b\right]$ is a zero-set: the existence of such an $x_{n}$ follows from the discussion already given. Then $T=\left\{x_{n}\right\}_{n<\omega}$ is as required.

COROLlary 6.3. Every closed interval of $X$ is an intersection of intervals that are zero-sets of $X$.

7. Linearly ordered $P$-spaces. The following theorem is an easy consequence of Theorem 4.2 .

Theorem 7.1. Let $X$ be any linearly ordered space. $A$ point $p \in X$ with character $\left\langle\rho, \sigma^{*}\right\rangle$ is a P-point if and only if both $\rho \neq \omega$ and $\sigma \neq \omega$.

Corollary 7.2. A linearly ordered space is a $P$-space if and only if every $\omega$-limit and every $\omega^{*}$-limit are gaps.

We can now present some simple examples.

EXAmple 7.3. In the compact space $W\left(\omega_{1}+1\right)(\S 6)$, every isolated point, and also the nonisolated end point $\omega_{1}$, are $P$-points; all remaining points of the space (i.e., the denumerable limit ordinals) are non- $P$-points. In the compact space $W\left(\omega_{2}+1\right)$, the $P$-points consist of the isolated points, the $\omega_{1}$-limits, and the end point $\omega_{2}$.

EXAMPLE 7.4. Let $X$ be any linearly ordered space, without gaps, every interval of which has the character set $\left\{c_{01}, c_{10}, c_{11}\right\}$ (Theorem 6.1). From Theorem 7.1, every symmetric element of $X$ is a $P$-point, and every nonsymmetric element is a non- $P$-point. Hence both the set $Y$ of all $P$-points, and its complementary set, are dense in the space. Furthermore, every point of $Y$ is a $P$-point of $Y$; thus $Y$ is a dense $P$-space.

EXAMPLE 7.5. Let $X$ be a dense linearly ordered space such that in every interval every point has the character $c_{21}$ and every gap has the character $c_{10}$ or $c_{00}$. Then $X$ is a $P$-space. From the fact that $\omega_{2}$ is not cofinal with $\omega_{1}$, it is clear that no point of $X$ can have a linearly ordered base of neighborhoods.

Example 7.6. Let $X$ be a dense linearly ordered space whose set of element characters is $\left\{c_{10}\right\}$ and whose set of gap characters is $\left\{c_{00}\right\}$. Then no point of $X$ is a $P$-point, yet every $Z(f)(f \in C(X, R))$, being a $G_{\delta}$, contains an open set.

Generalizations of these examples to higher cardinals are readily derived. 
The question of the existence of $P$-spaces of prescribed cardinality, however, is most easily settled by means of a direct construction, as follows.

THEOREM 7.7. (1) Let $A, B$ be disjoint nonempty closed subsets of an arbitrary $P$-space $X$ (not necessarily linearly ordered), for each of which every open covering has a subcovering of power $\leqq \aleph_{1}$; then $A, B$ can be separated by disjoint open sets. In particular, every $P$-space of power $\leqq \aleph_{1}$ is normal.

(2) For every cardinal $\mathfrak{l}>0$, there exists a discrete linearly ordered (hence normal) $P$-space of power $\mathfrak{t}$.

(3) For every $\mathfrak{m} \geqq \boldsymbol{\aleph}_{1}$, there exists a dense linearly ordered (hence normal) $P$-space of power $\mathrm{m}$.

(4) For every $\mathfrak{n} \geqq \boldsymbol{N}_{2}$, there exists a non-normal P-space of power $\mathfrak{n}$.

Proof. (1) This is established by a straightforward generalization of the standard proof that every regular separable space is normal $[14$, p. 102] (cf. also [19, p. 128]).

(2) Finite spaces are trivial. For every infinite order type $\beta$ the linearly ordered space of type $\left.{ }^{6}\right)\left(\omega^{*}+\omega\right) \beta$ is a discrete space of the same power as $\beta$.

(3) Consider first any regular $\omega_{\alpha}>\omega$, and put

$$
\pi_{\alpha}=\left(\omega^{*}+\omega\right)\left(\omega_{\alpha}^{*}+\omega_{\alpha}\right) .
$$

Let $A_{0}$ be any set of type $\pi_{\alpha}$. For every pair $D_{0, i}$ of consecutive elements of $A_{0}$, insert between these elements a new set $A_{1, i}$ of type $\pi_{\alpha}$, and put $A_{1}$ $=\cup_{i} A_{1, i}$. For each pair $D_{1, j}$ of consecutive elements of $A_{1}$, insert between them a new set $A_{2, j}$ of type $\pi_{\alpha}$, and put $A_{2}=\bigcup_{j} A_{2, j}$. Likewise define $A_{n}$ for all $n<\omega$, and, finally put $X=\mathrm{U}_{n<\omega} A_{n}$. Denote the order type of $X$ by $\rho_{\alpha}\left({ }^{7}\right)$. Since $\pi_{\alpha}$ is cofinal (coinitial) with $\omega_{\alpha}\left(\omega_{\alpha}^{*}\right)$, it is clear that every element of $X$ has the character $c_{\alpha \alpha}$; hence, using Corollary 7.2, $X$ is a dense linearly ordered $P$-space of power $\boldsymbol{\aleph}_{\alpha}$. To obtain a dense $P$-space $Y$ of arbitrary power $\boldsymbol{\aleph}_{\beta} \geqq \boldsymbol{\aleph}_{1}$, including singular $\boldsymbol{\aleph}_{\beta}$, one may take for the order type of $Y$ the type $\rho_{1} \omega_{\beta}$.

(4) Since $\rho_{\alpha}$ is cofinal with $\omega_{\alpha}$, any set of type $\rho_{\alpha}+1$ is also a $P$-space. To construct a non-normal $P$-space of power $\aleph_{2}$, let $X_{1}$ be of type $\rho_{1}+1$, and $X_{2}$ of type $\rho_{2}+1$. Denote their last elements by $b_{1}, b_{2}$, respectively. The product $X$ of the $P$-spaces $X_{1}$ and $X_{2}$ is a $P$-space of power $\boldsymbol{\aleph}_{2}$, and therefore so is its subspace $Y=X-\left\{\left\langle b_{1}, b_{2}\right\rangle\right\}$ (Corollaries 5.6 and 5.8). It follows from a classical argument due to Tychonoff [24] (a cofinality argument shows that the disjoint closed sets $\left\{b_{1}\right\} \otimes\left(X_{2}-\left\{b_{2}\right\}\right),\left(X_{1}-\left\{b_{1}\right\}\right) \otimes\left\{b_{2}\right\}$, cannot be separated) that $Y$ is not normal. Like constructions yield non-normal $P$-spaces of every regular cardinal $\geqq \boldsymbol{\aleph}_{2}$ (and of some singular cardinals). By taking the sum

( $)$ By the product $\sigma \tau$ is meant the order type of a set obtained by replacing each element of a linearly ordered set of type $\tau$ by a set of type $\sigma$.

(7) Thus $\rho_{\alpha}$ is the order type of the lexicographically ordered set of all finite sequences of elements of a set of type $\pi_{\alpha}$. 
of the above set $Y$ with an arbitrary $P$-space of any power $\boldsymbol{\aleph}_{\beta} \geqq \boldsymbol{\aleph}_{2}$, one obtains a non-normal $P$-space of power $\boldsymbol{\aleph}_{\beta}$ (singular $\boldsymbol{\aleph}_{\beta}$ included).

CoROllary 7.8. The continuum hypothesis is equivalent to the statement that every $P$-space of the power of the continuum is normal.

Corresponding equivalences are valid for $\omega_{\alpha}$-additive spaces [19] with $\alpha>1$.

REMARK 7.9. It has recently been shown by Henriksen and Isbell [9, Theorem 2] that if $X$ is any normal space, the residue class field $C(X, R) / M$ of any maximal ideal $M$ of $C(X, R)$ is always a real-closed field (for definition see $[26$, p. 235]). Actually, the proof is valid for any space $X$ in which every function continuous on a zero-set of $X$ can be extended continuously over $X$. In particular, then, the proof is valid if $X$ is a $P$-space (see (2) of Theorem 5.3). As we have just seen, a $P$-space need not be normal.

8. Introduction ( $Q$-spaces). An important problem in the study of rings of continuous functions is to ascertain when the ring determines the space. It has long been known that $C^{*}(X, R)$ determines $X$ if and only if $X$ is compact, and that $C(X, R)$ determines $X$ for a wider class of topological spaces, namely, Hewitt's $Q$-spaces.

Hewitt shows that for every maximal ideal $M$ of $C(X, R)$, the residue class field $C(X, R) / M$ is an ordered field containing the real field $R[10$, Theorem 41 ]. $M$ is called a real ideal if $C / M$ is $R$; otherwise (i.e., if $C / M$ is non-archimedean ordered) $M$ is called hyper-real. The maximal ideal $M$ is hyper-real if and only if there is a countable subfamily of $Z(M)$ whose total intersection is void [10, Theorem 50]. It is evident that if $M$ is fixed, $C / M$ is $R$.

Hewitt calls $X$ a $Q$-space if every maximal free ideal of $C(X, R)$ is hyperreal. Every completely regular space $X$ can be imbedded as a dense subset of a $Q$-space $v X$ (in fact $v X \subset \beta X$, whence $\beta v X=\beta X$ ), such that every function $f \in C(X, R)$ has a (unique) continuous extension over $v X\left(^{8}\right)$. Hence $C(X, R)$ is isomorphic with $C(v X, R)$. As mentioned above, two $Q$-spaces $X, Y$ are homeomorphic if and only if the rings $C(X, R), C(Y, R)$ are algebraically isomorphic [10, Theorem 57].

Shirota has shown [18, Theorem 1] that $X$ is a $Q$-space if and only if $X$ is homeomorphic with a closed subset of a product of real lines $\left({ }^{9}\right)$. It follows that every product of $Q$-spaces is a $Q$-space $\left({ }^{10}\right)$, and that every closed subset of a $Q$-space is a $Q$-space $\left({ }^{11}\right)$. According to a result obtained independently by Katětov and Shirota $[13$, Theorem $3 ; 18$, Theorem 3$]$, a paracompact space $X$ (definition below) is a $Q$-space if and only if every closed discrete subspace of $X$ is a $Q$-space.

$\left({ }^{8}\right)$ See also $[18 ; 5]$.

(9) The necessity was previously shown by Hewitt [10, Theorem 56$]$.

(10) This theorem was stated by Hewitt, but not correctly proved. We are indebted to Professor Hewitt for calling our attention to Shirota's result.

(11) An independent proof is given by Katětov [13, Proposition 4]. 
For the complete characterization of linearly ordered $Q$-spaces, we shall also need the concept of a nonmeasurable cardinal. A cardinal $\boldsymbol{\aleph}_{\alpha}$ will be called nonmeasurable $\left.{ }^{(12}\right)$ if no countably additive two-valued measure can be defined on the family of all subsets of a set of power $\boldsymbol{\aleph}_{\alpha}$, in such a way that every subset consisting of only one element has measure zero, and the entire set has measure one. It has been shown by Ulam and Tarski $[25 ; 23$, p. 153] that $\boldsymbol{\aleph}_{\alpha}$ is nonmeasurable for every $\boldsymbol{\aleph}_{\alpha}$ that is weakly accessible from $\boldsymbol{\aleph}_{0}$, i.e., smaller than the first strongly inaccessible cardinal $>\boldsymbol{N}_{0}\left({ }^{13}\right)$. It is known that the discrete space of power $\boldsymbol{\aleph}_{\alpha}$ is a $Q$-space if and only if the cardinal $\boldsymbol{\aleph}_{\alpha}$ is nonmeasurable [11, p. 175] (see also Theorem $10.4 \mathrm{ff}$., below).

It follows from these remarks that most paracompact spaces of current interest are $Q$-spaces. It is known, however, that there exist $Q$-spaces that are not normal, hence not paracompact $[10$, p. 94$]$.

A space $X$ is called paracompact (Dieudonné [3]) if every open covering of $X$ has a locally finite $\left({ }^{14}\right)$ refinement that covers $X$. Every metric space is paracompact [20], as is every locally compact space that is expressible as the union of countably many compact spaces [3].

We shall show that every linearly ordered space is countably paracompact $\left({ }^{15}\right)$, and that every linearly ordered $Q$-space is paracompact. These results are achieved by means of characterizations, in terms of the gaps of the space, of linearly ordered paracompact spaces (Theorem 9.5) and linearly ordered $Q$-spaces (Theorem 10.4). The latter leads, under a mild cardinality restriction, to a simple direct construction of $v X$ for any linearly ordered space $X$-it turns out that $v X$ is a subspace of a linearly ordered space.

\section{Linearly ordered paracompact spaces.}

Definition 9.1. Let $J$ be an interval of a linearly ordered space $X$. A gap $u$ of $J$ is covered in $J$ by an interval $K=(x, y)$ of $X$, if either $x<u<y$, or one of $x, y$ is an end-gap of $J$ and coincides with $u$. The gap $u$ is covered in $J$ by an open subset $U$ of $X$ if there is an interval $K$ of $X$, contained in $U$, such that $u$ is covered in $J$ by $K$. The gap $u$ is covered in $J$ by a family $U$ of open subsets of $X$, if it is covered in $J$ by some member of $U$.

If a gap $u$ of $J$ is covered in $X$ by $U$, then it is covered in $J$ by $U$. The converse need not hold, since an end-gap of $J$ may be an interior gap of $X$.

Lemma 9.2. Every open covering $\mathcal{T}$ of a linearly ordered space $E$, such that every gap of $E$ is covered (in $E$ ) by $\mathcal{T}$, has a finite subcovering.

(12) More precisely: non-two-valued-measurable.

(13) A regular cardinal $\boldsymbol{\aleph}_{\sigma}$ is strongly inaccessible if $\exp \boldsymbol{\aleph}_{\alpha}<\boldsymbol{\aleph}_{\sigma}$ for all $\alpha<\sigma$; see [22]. No such cardinal $>\boldsymbol{\aleph}_{0}$ is known to exist; any which might would be of almost incomprehensible magnitude. (Another proof of the Ulam-Tarski result can be derived from our Theorem 10.4 below.)

(14) A family $U$ of subsets of a space $X$ is locally finite if every point of $X$ has a neighborhood that meets only a finite number of sets of $U$.

(15) Dowker [4] has defined a space $X$ to be countably paracompact if every countable open covering of $X$ has a locally finite refinement that covers $X$. 
Proof. For each $T \in \mathcal{T}$, construct a subset $T^{\prime}$ of $E^{+}(\S 6)$, by adding to $T$ every gap of $E$ that is covered (in $E$ ) by $T$. Clearly, the family $\mathcal{T}^{\prime}=\left\{T^{\prime}\right\}_{T \in \mathcal{T}}$ is an open covering of $E^{+}$. Now it is well known that any linearly ordered space without gaps (including end-gaps) is compact (see the remark following Theorem 10.2 below). Therefore there is a finite subfamily $\left\{T_{k}^{\prime}\right\}$ of $\mathcal{T}^{\prime}$ that covers $E^{+}$. Obviously, the corresponding subfamily $\left\{T_{k}\right\}$ of $\tau$ covers $E$.

Fundamental to our main results are the notions of $Q$-sequence and $Q$-gap, which we now introduce.

Definition 9.3. Let $X$ be any linearly ordered space, and let $\omega_{\alpha}$ be any regular initial ordinal.

(1) An increasing or decreasing sequence $S=\left\{x_{\xi}\right\}_{\xi<\omega_{\alpha}}$ of points of $X^{+}$ (§6) is called a $Q$-sequence if for every nonzero limit ordinal $\lambda<\omega_{\alpha}$, the limit (in $X^{+}$) of the segment $\left\{x_{\xi}\right\}_{\xi<\lambda}$ of $S$ is a gap of $X$. In particular, every $\omega$-sequence and every $\omega^{*}$-sequence are $Q$-sequences.

(2) If $S$ is a $Q$-sequence, and if the gap $u$ is the limit of the entire sequence $S$, we speak of $S$ as a $Q$-sequence $a t u$.

(3) A gap $u$ is called a $Q-g a p$ from the left (right) if there exists an increasing (decreasing) $Q$-sequence at $u$, a nonmeasurable $Q$-gap from the left (right) if the cardinal of the $Q$-sequence is nonmeasurable.

(4) A gap $u$ is called a $Q$-gap if it is a $Q$-gap from both the left and the right (or only the appropriate one, in case $u$ is an end-gap), a nonmeasurable $Q$-gap if it is a nonmeasurable $Q$-gap from both the left and the right (or etc.).

It will be seen that the $Q$-sequences at gaps play.the role of discrete spaces, whereas the non- $Q$-sequences of any power $\boldsymbol{\aleph}_{\alpha}$ behave essentially like the space of ordinals $W\left(\omega_{\alpha}\right)$. For denumerable $Q$-sequences, these roles coincide.

Let $U$ be any open subset of a linearly ordered space $X$. For any point $p$ of $U$, the union of all the intervals of $X$ contained in $U$ and containing $p$ is an interval of $X$, which we shall call a maximal interval $\left({ }^{16}\right)$ of $U$. Clearly, distinct maximal intervals are disjoint.

Lemma 9.4. Let $J=(p, v)$ be an interval of a linearly ordered space $X$, where $v$ is a gap that is not a $Q$-gap from the left. Let $\alpha$ be the ordinal for which $v$ is an $\omega_{\alpha}$-limit. Let $\mathcal{T}$ be any open covering of $J$ that does not cover the gap $v$. Then $\mathcal{T}$ has a subfamily of power $\boldsymbol{\aleph}_{\alpha}$ with nonvoid intersection. In particular, $\mathcal{\tau}$ is not locally finite.

Proof. We may suppose that every $T \in \mathcal{T}$ is a subset of $J$. Then every maximal interval of $T$ is an open subinterval $K=(x, z)$ of $J$. Denote by $K$ the family, over all $T \in \mathcal{T}$, of all these maximal intervals $K$. Since the gap $v$ is not covered, we have $z<v$ for every $K=(x, z) \in K$. Now let $\left\{y_{\xi}\right\}_{\xi<\omega_{\alpha}}$ be any increasing sequence of points of $J$ whose limit is $v$. Each point $y_{\xi}$ is covered by at least one $T \in \mathcal{T}$, hence by at least one $K \in K$; and for every $T, y_{\xi}$ is covered by at most one maximal interval $K$ of $T$. Let $K_{\xi}$ denote the family

${ }^{(16)}$ If $X$ is connected, the maximal intervals of $U$ are the same as the components of $U$. 
of all intervals $K \in K$ that cover $y_{\xi}$. Define $K_{\xi}=\left(x_{\xi}, z_{\xi}\right)=\bigcup_{K \in K_{\xi}} K$. Now assume the lemma to be false. Then the cardinal of $K_{\xi}$ must be $<\boldsymbol{\aleph}_{\alpha}$. But $z<v$ for every $K=(x, z) \in K$; therefore, since $v$ is an $\omega_{\alpha}$-limit (whence, according to our definition, $\omega_{\alpha}$ is regular), we have $z_{\xi}<v$. Since $y_{\xi}<z_{\xi}$, it is a straightforward matter to construct an increasing sequence

$$
S=\left\{y_{\sigma_{0}}, z_{\sigma_{0}}, y_{\sigma_{1}}, z_{\sigma_{1}}, \cdots, y_{\sigma_{\xi}}, z_{\sigma_{\xi}}, \cdots\right\} \quad\left(\xi<\omega_{\alpha}\right) .
$$

The limit of this sequence is $v$, so by hypothesis $S$ cannot be a $Q$-sequence. Accordingly, there is a point $y^{\prime} \in J$ that is the limit of a segment of $S$ of some limit type $\lambda$. Let $K^{\prime}=\left(x^{\prime}, z^{\prime}\right)$ be any member of $K$ that covers $y^{\prime}$. Then $x^{\prime}<y^{\prime}<z^{\prime}$. Since $y^{\prime}=\lim _{\xi<\lambda} y_{\sigma_{\xi}}$, there is an ordinal $\eta<\lambda$ such that $x^{\prime}<y_{\sigma_{\eta}}<y^{\prime}$. Then $y_{\sigma_{\eta}} \in K^{\prime}$. Consequently $K^{\prime} \in K_{\sigma_{\eta}}$ (by definition of the latter). Therefore $K^{\prime} \subset K_{\sigma_{\eta}}$, i.e., $\left(x^{\prime}, z^{\prime}\right) \subset\left(x_{\sigma_{\eta}}, z_{\sigma_{\eta}}\right)$. But this is impossible, since the relation $y^{\prime}=\lim _{\xi<\lambda} z_{\sigma_{\xi}}$ implies that $z_{\sigma_{\eta}}<y^{\prime}$, whence $z_{\sigma_{\eta}}<z^{\prime}$.

The theorem that follows is one of our main results.

Theorem 9.5. A linearly ordered space $X$ is paracompact if and only if every gap of $X$ is a Q-gap. On the other hand, every linearly ordered space is countably paracompact.

Proof. Suppose first that there is a gap $v$, not the left end-gap, say, that is not a $Q$-gap from the left. Let $\left\{y_{\xi}\right\}_{\xi<\omega_{\alpha}}$ be any increasing sequence of points of $X^{+}(\S 6)$ whose limit is $v$, in which $y_{0}$ is the first point or gap of $X$. Let $w$ denote the last point or gap of $X$. Let the open covering $U$ of $X$ consist of all the intervals $\left[y_{0}, y_{\xi}\right), 0<\xi<\omega_{\alpha}$, and in addition, if $v<w$, the interval $(v, w]$. Let $\mathcal{T}$ be an arbitrary refinement of $U$ that covers $X$. Then the gap $v$ is not covered by $\mathcal{\tau}$. Hence by Lemma $9.4, \mathcal{T}$ cannot be locally finite. Therefore $X$ is not paracompact.

For the remainder of the proof, let $U$ be an arbitrary open covering of $X$, and suppose that either $U$ is countable or every gap of $X$ is a $Q$-gap. We are to find a locally finite refinement of $U$ that covers $X$. Denote by $F^{+}$the set of all gaps of $X$ that are not covered in $X$ by $U$. Clearly, every limit-point (in $X^{+}$) of non-covered gaps is a non-covered gap; consequently $F^{+}$is a closed subset of $X^{+}$. Its complement in $X^{+}$is therefore expressible as the union of disjoint intervals $K^{+}$open in $X^{+}$. Each boundary point of any such interval $K^{+}$is either a non-covered gap, or an end point of $X^{+}$. The corresponding intervals $K=K^{+} \cap X$ are disjoint open (and closed) intervals of $X$, whose union is $X$. We may accordingly deal with each such $K$ independently: for every $U \in U$ define $U_{K}=U \cap K$; it is sufficient to find a locally finite refinement of the family $U_{K}=\left\{U_{K}\right\}_{U \in U}$ that covers $K$.

Consider then any fixed $K$. Write $K=[u, v]$. Let $p$ be any interior point of $K$, and put $H=[u, p], J=[p, v]$. If there exist locally finite refinements $\mathscr{W}_{H}$ and $\mathfrak{W}_{J}$ of $U_{K}$, covering $H$ and $J$, respectively, then there exists a locally finite refinement of $V_{K}$ that covers $K$ : this follows readily from the fact that the point $p$ is covered by only finitely many maximal intervals of the open sets 
belonging to the families $\mathfrak{W}_{H}$ and $\mathfrak{W}_{J}$.

The problem is accordingly reduced to that of finding a locally finite refinement $W_{J}$ of $U_{K}$ that covers the interval $J=[p, v]$. Now by construction of $K$, every interior gap of $J$ is covered by $U$, and hence by $U_{K}$. Consequently if $v$ itself is covered by $U_{K}$, then the existence of the desired refinement is provided by Lemma 9.2. Assume, then, that $v$ is a gap and that $v$ is not covered by $U_{K}$.

According to our hypotheses, either $v$ is a $Q$-gap or $U_{K}$ is countable. Then in any case $v$ must be a $Q$-gap from the left. For suppose that there is no increasing $Q$-sequence at $v$. Then $v$ is an $\omega_{\alpha}$-limit for some $\alpha>0$. But Lemma 9.4 requires that $U_{K}$ contain a subfamily of power $\boldsymbol{\aleph}_{\alpha}$, so $U_{K}$ cannot be countable.

Let then $S=\left\{y_{\xi}\right\}_{\xi<\omega_{\alpha}}$ be an increasing $Q$-sequence at $v$. We may certainly take $y_{0}=p$, and we may suppose that every term $y_{\xi+1}$ is a point of $X$. We may assume further that the limits of the segments of $S$ have been incorporated into the sequence, so that $y_{\lambda}=\lim _{\xi<\lambda} y_{\xi}$ for every nonzero limit ordinal $\lambda<\omega_{\alpha}$; since $S$ is a $Q$-sequence, each such term $y_{\lambda}$ is a gap.

Next, for each $\xi<\omega_{\alpha}$, define the closed interval $E_{\xi}=\left[y_{\xi}, y_{\xi+1}\right]$. Recall now that the only gap of $J$ that is not covered by $U_{K}$ is its end-gap $v$. Hence, for every $\xi<\omega_{\alpha}$, every gap of $E_{\xi}$ is covered by $U_{K}$. By Lemma 9.2, there is a finite subfamily $\mathfrak{W}_{\xi}$ of $\bigcup_{K}$ that covers $E_{\xi}$. Since any two successive intervals $E_{\xi}, E_{\xi+1}$ have just one point in common, and since $y_{\lambda}$ is a gap for every nonzero limit ordinal $\lambda<\omega_{\alpha}$, we can easily refine every family $\mathscr{W}_{\xi}$ to a family $W_{\xi}^{\prime}$ having the following properties:

(1) (a) for every $\xi<\omega_{\alpha}, W_{\xi}^{\prime} \cup W_{\xi+1}^{\prime} \cup W_{\xi+2}^{\prime}$ is a finite open covering of $E_{\xi+1}$;

(b) for every limit ordinal $\lambda\left\langle\omega_{\alpha}, W_{\lambda}^{\prime} \cup \mathscr{W}_{\lambda+1}^{\prime}\right.$ is a finite open covering of $E_{\lambda}$;

(2) for every $\xi<\omega_{\alpha}$, and every $\eta \geqq \xi+2, W_{\xi} \cap W_{\eta}=0$ for all $\mathscr{W}_{\xi} \in W_{\xi}^{\prime}$ and all $W_{\eta} \in \mathscr{W}_{\eta}^{\prime}$. The family $\mathscr{W}_{J}=\cup_{\xi<\omega_{\alpha}} \mathscr{W}_{\xi}^{\prime}$ is then a locally finite refinement of $V_{K}$ which covers $J$. This completes the proof of the theorem.

One might define a family of subsets of $X$ to be "locally $<\boldsymbol{\aleph}_{\beta}$ " if every point of $X$ has a neighborhood that meets $\left\langle\boldsymbol{\aleph}_{\beta}\right.$ sets of the family. One can then find theorems relating this number $\boldsymbol{\aleph}_{\beta}$ to the cardinal number of an open covering and the cardinal numbers of the non- $Q$-sequences, from which Theorem 9.5 would appear as a corollary. For example, Theorem 9.5 shows that the space $W\left(\omega_{1}\right)(\S 6)$ is not paracompact, whereas the proof shows, in fact, that there is an open covering of $W\left(\omega_{1}\right)$ having no "locally denumerable" refinement that covers $W\left(\omega_{1}\right)$. These questions are not our primary concern, however, and we leave the subject at this point without further comment, turning our attention now to $Q$-spaces.

\section{Linearly ordered $Q$-spaces.}

Definition 10.1. Let $X$ be any linearly ordered space, and let $u$ be any gap of $X$. Any interval of $X$ whose right (left) boundary is $u$ will be called a left (right) interval at $u$. The ideal of $C(X, R)$ consisting of all functions that vanish on a left (right) interval at $u$ is denoted by $L_{u}\left(R_{u}\right)$. (It is understood 
here and subsequently that $u$ is not the left (right) end-gap of $X$.)

Theorem 10.2. Let $X$ be any linearly ordered space. For every gap $u$ of $X$ the ideals $L_{u}$ and $R_{u}$ of $C(X, R)$ are free (hence every maximal ideal containing $L_{u}$ or $R_{u}$ is free). On the other hand, if $M$ is any maximal free ideal of $C(X, R)$, then there exists (exactly) one gap $u$ of $X$ such that either $M \supset L_{u}$ or $M \supset R_{u}$.

Proof. For the first part of the proof, it suffices to consider the case of $L_{u}$. From Lemma 6.2, there is an increasing sequence $\left\{x_{\xi}\right\}$, with limit $u$, such that every interval $\left[x_{\xi}, u\right]$ is a zero-set. Obviously these are all in $Z\left(L_{u}\right)$, and their intersection is empty. Thus $L_{u}$ is a free ideal.

For the remainder of the proof, let $a$ denote the first element or gap of $X$ and $b$ the last. Then $[a, b]=X \in Z(M)$. Put

$$
A=\left\{y \mid y \in X^{+},[a, y] \in \mathcal{Z}(M)\right\}, \quad B=\left\{x \mid x \in X^{+},[x, b] \in \mathcal{Z}(M)\right\},
$$

and define $v=\inf A, w=\sup B\left(v \in X^{+}, w \in X^{+}\right)$. We shall show first that $w=v$. If $v<w$, then by definition of $v$ there exists a $y \in A$ such that $y<w$; in turn there exists an $x \in B$ such that $y<x$. Then the zero-sets $[a, y],[x, b]$ of $Z(M)$ are disjoint, which is impossible. Suppose now that $w<v$. We define $s$ and $t$ as follows. If $w, v$ are consecutive elements of $X$, put $s=v, t=w$. If not, let $u$ be any point such that $w<u<v$. Lemma 6.2 implies that there is an $s$, with $w<s \leqq u$, such that the closed interval $[s, b]$ is a zero-set. Likewise there is a zero-set $[a, t]$, for some $t$ with $u \leqq t<v$. Hence in either case the intervals $[a, t]$ and $[s, b]$ are zero-sets, and their union is $X$. Therefore one of them is in $Z(M)$. Hence $t \in A$ or $s \in B$. But each of these is impossible, since $t<v$ and $s>w$.

Hence, finally, we must have $w=v$. Now by the finite intersection property, for every $x \in B$ and $y \in A$ the set $[a, y] \cap[x, b]=[x, y]$ is in $Z(M)$. Thus if $v$ is a point of $X$, then every $Z$ in $Z(M)$ meets every neighborhood of $v$, whence $Z$, being a closed set, contains $v$; in this event the ideal $M$ is fixed, not free. It follows that $v$ is a gap. Then each of the disjoint intervals $[a, v]$, $[v, b]$ is open and closed, hence a zero-set, and therefore one of them, say $[a, v]$, is in $Z(M)$. Then for every $x$ in $B$ the interval $[x, b] \cap[a, v]=[x, v]$ is in $\mathcal{Z}(M)$. Hence $M \supset L_{v}$.

It follows from this theorem that $C$ contains a free ideal if and only if $X$ has a gap. Since $C$ contains a free ideal if and only if $X$ is noncompact ( $\$ 2$ ), we obtain the familiar result that a linearly ordered space is compact if and only if it has no gaps.

ThEOREM 10.3. Let $X$ be any linearly ordered space, and let $u$ be any gap of $X$ other than the left end-gap.

(1) If $u$ is not a Q-gap from the left, then the ideal $L_{u}$ is a real maximal free ideal.

(2) If $u$ is a nonmeasurable Q-gap from the left, then every maximal (free) ideal containing $L_{u}$ is hyper-real. 
Proof. (1) We have already shown in Theorem 10.2 that in any case the ideal $L_{u}$ is free. Since no increasing $Q$-sequence exists at $u, u$ is an $\omega_{\alpha}$-limit for some $\alpha>0$. Therefore every countable intersection of left intervals at $u$ is nonempty. As pointed out above, a maximal ideal is real if (and only if) every countable intersection of its zero-sets is nonempty. It follows that if $L_{u}$ is maximal, then it is real.

Let $M$ be any maximal ideal containing $L_{u}$, and consider an arbitrary $f \in M$. We show that $f \in L_{u}$, which will complete the proof. Since $Z(f)$ meets every member of $Z\left(L_{u}\right)$, there is an increasing sequence $S=\left\{x_{\xi}\right\}_{\xi<\omega_{\alpha}}$ of zeros of $f$, with limit $u$. Now let $n$ be any positive integer, and denote by $Y_{n}$ the set of points $y<u$ at which $|f(y)| \geqq 1 / n$; we shall see that if $Y_{n}$ is not empty, then $\sup Y_{n}<u$. In fact, in the contrary case, there exists a sequence $T_{n}$ $=\left\{y_{\xi}\right\}_{\xi<\omega_{\alpha}}$, of points of $Y_{n}$, with limit $u$. Since $\omega_{\alpha}$ is regular, it is an elementary matter to construct an increasing sequence

$$
\left\{x_{0}^{\prime}, y_{0}^{\prime}, x_{1}^{\prime}, y_{1}^{\prime}, \cdots, x_{\xi}^{\prime}, y_{\xi}^{\prime}, \cdots\right\}
$$

in which every $x_{\xi}^{\prime} \in S, y_{\xi}^{\prime} \in T_{n}$. The limit of this sequence is of course $u$. By hypothesis, therefore, it cannot be a $Q$-sequence, so it has at least one segment whose limit is a point $z$ of $X$. But then by continuity of $f$, we have both $f(z)=0$ and $|f(z)| \geqq 1 / n$. Put $w_{n}=\sup Y_{n}$; it follows from the contradiction just established that we must have $w_{n}<u$. Write $w=\lim _{0<n<\omega} w_{n}$; since $\omega_{\alpha}$ is not cofinal with $\omega$, we have $w<u$. Therefore $f$ vanishes on the interval $[w, u]$, i.e., $f \in L_{u}$.

(2) Let $M$ be any maximal free ideal such that $M \supset L_{u}$. Let $\alpha$ be the ordinal for which $u$ is an $\omega_{\alpha}$-limit. If $\alpha=0$, we are finished: by Lemma 6.2, there is a countable subfamily of $\mathcal{Z}\left(L_{u}\right)$, hence of $\mathcal{Z}(M)$, with void intersection, whence $M$ is hyper-real. Assume then that $\alpha>0$. Take any increasing $Q$-sequence $S$ at $u$, and let $\left\{u_{\xi}\right\}_{\xi<\omega_{\alpha}}$ denote the increasing sequence of gaps constituting the limits, other than $u$ itself, of the set $S$. The intervals $J_{\xi}=\left(u_{\xi}, u_{\xi+1}\right)$ are open and closed, and their union $J=\bigcup_{\xi<\omega_{\alpha}} J_{\xi}$ is an open and closed left interval at $u$, whence $J \in Z(M)$. Consequently, if $A, B$ are any two complementary subsets of the set of ordinals $W\left(\omega_{\alpha}\right)$, then each of the complementary subsets $J_{A}=\bigcup_{\xi \in A} J_{\xi}, J_{B}=\bigcup_{\eta \in B} J_{\eta}$, of $J$, is open and closed, and exactly one of them is in $\mathcal{Z}(M)$. Now denote by $\mathcal{F}$ the set of intervals $\left\{J_{\xi}\right\}_{\xi<\omega_{\alpha}}$; every subset of $\mathcal{F}$ is of the form $\mathcal{F}_{A}=\left\{J_{\xi}\right\}_{\xi \in A}$. Define a finitely additive two-valued measure $m$ on the family of all subsets of $\mathcal{F}$, by putting $m\left(\mathcal{f}_{A}\right)=1$ if $J_{A} \in \mathcal{Z}(M)$, $m\left(\mathcal{f}_{A}\right)=0$ if $J_{A} \in \mathcal{Z}(M)$. Since $M \supset L_{u}$, it is obvious that "points" have measure zero (i.e., for each $\xi<\omega_{\alpha}$, we have $J_{\xi} \notin \mathcal{Z}(M)$ ). Moreover, $m(\mathcal{f})=1$. Now since by hypothesis $\boldsymbol{\aleph}_{\alpha}$ is a nonmeasurable cardinal, the measure $m$ cannot be countably additive. Hence there exists a countable family $\left\{\mathcal{F}_{A_{n}}\right\}$ $(n=1,2, \cdots)$ of subsets of $\mathcal{F}$ of measure one, whose intersection $\mathcal{F}_{A^{\prime}}$ is of measure zero. Then $J_{A^{\prime}} \in \mathcal{Z}(M)$. Therefore $J-J_{A^{\prime}} \in Z(M)$. Hence $\left\{J_{A_{n}}\right.$ $\left.\cap\left(J-J_{A^{\prime}}\right)\right\}(n=1,2, \cdots)$ is a countable family of zero-sets of $M$ having empty intersection. Thus $M$ is hyper-real. This completes the proof of the theorem. 
Remark. For the special case in which $\boldsymbol{\aleph}_{\alpha} \leqq \exp \boldsymbol{\aleph}_{0}$, the following alternate to the last part of the proof may be of interest. Let $\left\{r_{\xi}\right\}_{\xi<\omega_{\alpha}}$ be any set of $\boldsymbol{\aleph}_{\alpha}$ distinct nonzero real numbers, and define a function $f$ as follows: $f=r_{\xi}$ on $J_{\xi}$ (for all $\left.\xi<\omega_{\alpha}\right), f=0$ on $X-J$. Then $f \in C(X, R)$. If now $M$ is real, let $r$ denote that real number for which $f-r \in M$; then either $r=0$, or $r=r_{\xi}$ for some $\xi$. Each of these is impossible, however, since neither $X-J$ nor any $J_{\xi}$ is in $\mathcal{Z}(M)$.

The preceding theorem provides us with a large class of maximal free ideals that can be explicitly described. An easy example is the ideal $L_{\omega_{1}}$ in the space of ordinals $W\left(\omega_{1}\right)$, that is, the ideal consisting of all functions each of which vanishes from some point on. A somewhat more interesting example may be constructed as follows. Let $T$ be a dense linearly ordered space without gaps, whose set of characters is $\left\{c_{00}, c_{11}\right\}$. Delete any $c_{11}$-element $u$ from $T$, and call the resulting space $S$. Clearly there is no $Q$-sequence at the gap $u$ of $S$. The ideals $L_{u}$ and $R_{u}$ (both exist if $u$ is an interior gap) are therefore maximal ideals. Again, let $Y$ be a dense linearly ordered space whose set of gap characters is $\left\{c_{00}\right\}$ and whose set of element characters is $\left\{c_{11}, c_{22}\right\}$. Construct $X$ by deleting any $c_{22}$-point $u$; then $L_{u}$ and $R_{u}$ are maximal free ideals. In this example, $X$ is a $P$-space (cf. Example 10.10).

We are now ready to obtain our characterization of linearly ordered $Q$ spaces.

THEOREM 10.4. For any linearly ordered space $X$, the following statements are equivalent.

(1) $X$ is a $Q$-space.

(2) $X$ is paracompact, and every closed subspace of $X$ is a $Q$-space.

(3) $X$ is paracompact, and every closed discrete subspace of $X$ is a $Q$-space.

(4) Every gap of $X$ is a nonmeasurable Q-gap.

Proof. (1) implies (2). If there is gap of $X$ that is not a $Q$-gap, then by Theorem 10.3 there is a maximal free ideal of $C(X, R)$ that is not hyper-real. Hence if $X$ is a $Q$-space, then every gap is a $Q$-gap; therefore, by Theorem 9.5, $X$ is paracompact. The result now follows upon recalling that every closed subspace of a $Q$-space is a $Q$-space.

(2) implies (3). Trivial. Before continuing we remark that according to the result of Katětov and Shirota, quoted above, (3) implies (1) for arbitrary spaces, not only for those that are linearly ordered.

(3) implies (4). If $X$ is paracompact, then every gap is a $Q$-gap (Theorem 9.5). Hence it suffices to show that if every closed discrete subspace of $X$ is a $Q$-space, then every $Q$-gap is nonmeasurable. Let $S$ be any $Q$-sequence at a gap $u$, in which every term of $S$ is a point of $X$. Then $S$ is a closed discrete subspace of $X$. Therefore, by hypothesis, $S$ is a $Q$-space. Let $m$ be any finitely additive two-valued measure defined on all subsets of $S$, with $m(S)=1$, and 
such that points have measure zero. The family $\mathcal{Z}$ of all sets of measure one is closed under finite intersection and under extension. Moreover, if a subset $Z^{\prime}$ of $S$ meets every $Z \in Z$, then $Z^{\prime} \in Z$ (for if $m\left(Z^{\prime}\right)=0$ then $m\left(S-Z^{\prime}\right)=1$, i.e., $S-Z^{\prime} \in \mathcal{Z}$ ), and therefore, finally, $Z$ has total intersection void (since any point common to all $Z \in Z$ would be in $Z$ and hence have measure one). It follows that $Z=Z(M)$ for some maximal free ideal $M$ of $C(S, R)$ (see §2). Since $S$ is a $Q$-space, $M$ is hyper-real, so there is a countable subfamily of $\mathcal{Z}(M)$ with void intersection. Hence the measure $m$ is not countably additive. Therefore the cardinal of $S$ is nonmeasurable.

(4) implies (1). This is an immediate consequence of Theorems 10.2 and 10.3. Our proof is now complete.

Since every discrete space can be regarded as linearly ordered (Theorem 7.7), the arguments given above yield an alternate proof of the known fact $\left[11\right.$, p. 175] that the discrete space of power $\boldsymbol{\aleph}_{\alpha}$ is a $Q$-space if and only if the cardinal $\boldsymbol{\aleph}_{\alpha}$ is nonmeasurable.

\section{COROLlaRy 10.5. Every linearly ordered Q-space is paracompact.}

THEOREM 10.6. Let $X$ be any linearly ordered space, and let $u$ be any gap of $X$ other than the left end-gap. Then $u$ is a non-Q-gap from the left if and only if every function $f \in C(X, R)$ is constant on a left interval at $u$.

Proof. If no increasing $Q$-sequence exists at $u$, then $L_{u}$ is a real maximal ideal (Theorem 10.3). Hence for every $f \in C$, there is a real number $r$ such that $f-r \in L_{u}$, so $f=r$ on a left interval at $u$.

Conversely, let there exist an increasing $Q$-sequence at $u$, of type $\omega_{\alpha}$. Suppose first that $\alpha>0$. Let the family of intervals $\left\{J_{\xi}\right\}_{\xi<\omega_{\alpha}}$ be as in the proof of Theorem 10.3. The function $f$ that is 0 on every $J_{\xi}$ with even index $\xi$, and 1 on every one with odd index (and, say, 0 on $X-J$ ), is continuous. Suppose, finally, that $\alpha=0$. From Lemma 6.2, there is an increasing sequence $\left\{x_{n}\right\}_{n<\omega}$ such that every interval $K_{n}=\left[x_{n}, u\right]$ is a zero-set. If $K_{n}=Z\left(f_{n}\right)$, then also $K_{n}=Z\left(g_{n}\right)$, where $g_{n}=\min \left\{\left|f_{n}\right|, 1\right\}$. Define $g=\sum_{n=1}^{\infty} 2^{-n} g_{n}$; then $g$ is continuous. And $g$ cannot be constant on any left interval at $u$, for on any interval $K_{n}$, we have $0<g<2^{-n}$.

If $X$ is any linearly ordered space for which the cardinal of every $Q$-sequence whose limit is a gap is nonmeasurable, then $v X(\S 8)$ can be described very simply.

First construct a space $X^{\prime \prime}$ without gaps by replacing every gap $u$ of $X$ by two new elements, $l_{u}$ and $r_{u}$ (or only the appropriate one, in case $u$ is an end-gap). The space $X^{\prime \prime}$ is to be ordered in the natural way, preserving the order of $X$ : for every gap $u$ of $X$, the new elements $l_{u}, r_{u}$ are consecutive elements of $X^{\prime \prime}$, with $l_{u}<r_{u}$, and for all $x \in X$, both $l_{u}$ and $r_{u}$ bear the same order relation to $x$ as does the gap $u$. Then $X$ is a dense subspace of the linearly ordered space $X^{\prime \prime}$. Next construct an intermediate space $X^{\prime}$, by deleting every 
element $l_{u}$ for which the gap $u$ of $X$ is a $Q$-gap from the left, and every $r_{u}$ for which $u$ is a $Q$-gap from the $\operatorname{right}\left({ }^{17}\right)$. Now by Theorems 10.2 and 10.3 , a maximal free ideal $M$ of $C(X, R)$ is real if and only if $M=L_{u}$ or $M=R_{v}$, fci some gap $u(v)$ of $X$ that is a non- $Q$-gap from the left (right). Thus there is a one-one correspondence between the real maximal free ideals of $C(X, R)$ and the points of $X^{\prime}-X$. Moreover, it follows at once from Theorem 10.6 that every $f \in C(X, R)$ can be continuously extended over $X^{\prime}$. Finally, $X$ is dense in $X^{\prime}$. But these are the three characteristic properties of the construction of $v Y$ for an arbitrary space $Y[10$, Theorem $58 ; 5]$. We have proved:

Theorem 10.7. Let $X$ be any linearly ordered space, and let $X^{\prime}$ be the corresponding space described above; then $X^{\prime}$ is $v X$ (under the cardinaliiy restriction noted).

It can be seen without difficulty that if $\mathfrak{m}$ is the cardinal of a linearly ordered set $X$, then the cardinal of the set of gaps of $X$ is at most exp m. Hence:

Corollary 10.8. If $\mathfrak{m}$ is the cardinal of a linearly ordered space $X$, then the cardinal of $v X$ is at most exp $\mathfrak{m}$ (under the cardinality restriction noted above).

We conclude with some examples.

Example 10.9. Let $X$ be a dense linearly ordered space whose set of element characters is $\left\{c_{11}\right\}$ and whose set of gap characters is $\left\{c_{00}, c_{22}\right\}$, the $c_{22^{-}}$ gaps being everywhere dense. Then the set of $Q$-gaps of $X$, and the set of non- $Q$-gaps, are each everywhere dense. (Also, this space is a $P$-space.)

EXAMPLE 10.10. Let $Y$ be a dense linearly ordered space whose set of gap characters is $\left\{c_{00}\right\}$ and whose set of element characters is $\left\{c_{11}, c_{22}\right\}$. Then $Y$ is paracompact and a $Q$-space (and a $P$-space). Now delete a single $c_{22}-$ element $u$. The resulting space $X$ is no longer paracompact, nor is it a $Q$-space. But it remains a $P$-space, and therefore, like $Y$, it is not countably compact. If $u$ is an end-gap of $X$, then $v X$ is $Y$. If $u$ is an interior gap, then two new points must be inserted to yield $v X$. In either case, however, the cardinal number of $\beta X-X$ is at least exp exp $\boldsymbol{\aleph}_{0}$ (since $X$ is normal but not countably compact; see $[2 ; 17])$.

EXAmPLe 10.11. Let $Y$ be a dense linearly ordered space, without gaps, whose set of characters is $\left\{c_{00}, c_{10}\right\}$. Then $Y$ is a $Q$-space (in fact, $Y$ is compact). Construct $X$ by deleting a $c_{10}$-element $u$. Let $(A \mid B)$ be the Dedekind cut of $X$ that is the gap $u$ (thus $Y=A+\{u\}+B$ ). Construct $X^{\prime \prime}$ by replacing the gap $u$ by new elements $l_{u}$ and $r_{u}$, as in the discussion preceding Theorem 10.7. Then $v A=A+\left\{l_{u}\right\}$, and $X^{\prime}=v X$ is the sum of the two $Q$-spaces $A+\left\{l_{u}\right\}$ and $B$. Thus, here, $v X$ is not a linearly ordered space, although it is a subspace of the linearly ordered space $X^{\prime \prime}$.

Remarks (added November 4, 1953).

(17) In general, $X^{\prime}$ will not be a linearly ordered space. See Footnote 5 and Example 10.11. 
1. (§3.) The proof of Theorem 3.6 shows that if the maximal ideal $M^{p}$ is hyper-real, then $p$ is not a $P$-point of $\beta X$. Hence if $X$ is a $Q$-space, then no point of $\beta X-X$ is a $P$-point of $\beta X$. (The converse is false, as is shown by the example of the space $W(\omega+1) \otimes W\left(\omega_{1}+1\right)-\left\{\left\langle\omega, \omega_{1}\right\rangle\right\}$.) As in Remark 3.7, we obtain as a corollary: if $X$ is a $Q$-space, then the number of nonmaximal prime ideals of $C^{*}(X, R)$ is at least the cardinal of $\beta X-X$. Remark 3.7 itself is a special case of this corollary, since if $X$ is the discrete space of power $\boldsymbol{\aleph}_{\alpha}$, then $\beta X$ is of power $\exp \exp \boldsymbol{\aleph}_{\alpha}[17]$.

2. (\$7.) An example of a nondiscrete linearly ordered $P$-space was given by Dieudonné in Notes de tératopologie-I, Revue Scientifique vol. 77 (1939) pp. 39-40.

3. (Remàrk 7.9.) Isbell has now found a proof (to be published shortly) that $C(X, R) / M$ is a real-closed field for arbitrary completely regular $X\left({ }^{18}\right)$.

4. (Theorem 9.5.) A family of subsets of $X$ is called point-finite if every point meets only finitely many members of the family, star-finite if every member of the family meets only finitely many others. If, in the definition of paracompact space, we replace locally finite by point-finite resp. star-finite, we obtain the definition of metacompact space resp. space with the star-finite property. Clearly, every space with the star-finite property is paracompact, and every paracompact space is metacompact. A trivial modification of our proof of Theorem 9.5 (making use of the stronger of the two conclusions of Lemma 9.4) shows that for linearly ordered spaces, these three concepts coincide.

5. (Theorem 9.5.) The result that every linearly ordered space is countably paracompact has been announced independently by B. J. Ball (Bull. Amer. Math. Soc. Abstract 59-5-553)( $\left.{ }^{19}\right)$.

6. $(\S \S 9,10$.) Observe that the number of gaps of a linearly ordered space is not a topological invariant. For example, the denumerable discrete space can be represented as the space of positive integers, or of all integers, or as the order type $\left(\omega^{*}+\omega\right) \eta(\eta=$ order type of the rationals); these spaces have, respectively, 1 gap, 2 gaps, exp $\boldsymbol{\aleph}_{0}$ gaps.

7. (Corollary 10.5.) The question is naturally raised as to whether every normal $Q$-space (not necessarily linearly ordered) is paracompact. The answer is no: Professor R. H. Bing has communicated to us a proof that the space constructed in Example $\mathrm{H}$ of his paper Metrization of topological spaces (Canadian Journal of Mathematics vol. 3 (1951) pp. 175-186) is a normal $Q$-space but is not paracompact. We omit the proof here.

Added in proof. We have since proved the following theorem. Let $X$ be a normal $P$-space. Then (A): for every noncompact closed subset $Y$ of $X$, there exists a function $f$ in $C(X, R)$ such that $f$ is unbounded on $Y$. For the proof, see L. Nachbin, Topological vector spaces of continuous functions, Proc. Nat. Acad.

(18) Added in proof. Proc. Amer. Math. Soc. vol. 5 (1954) p. 439.

(19) Added in proof. Proc. Amer. Math. Soc. vol. 5 (1954) pp. 190-192. 
Sci. U.S.A. vol. 40 (1954) pp. 471-474, footnote 8. In this paper, it is shown that if $X$ is not a $Q$-space but satisfies (A) (e.g., Example 10.9), then $C(X, R)$, in the compact-open topology, is a $t$-space that is not bornological. (This was also proved by T. Shirota, On locally convex vector spaces of continuous functions, Proc. Japan Acad. vol. 30 (1954) pp. 294-298.)

\section{REFERENCES}

1. R. Arens and I. Kaplansky, Topological representations of algebras, Trans. Amer. Math. Soc. vol. 63 (1948) pp. 457-481.

2. E. Cech, On bicompact spaces, Ann. of Math. vol. 38 (1937) pp. 823-844.

3. J. Dieudonné, Une génêralisation des espaces compacts, J. Math. Pures Appl. vol. 23 (1944) pp. 65-76.

4. C. H. Dowker, On countably compact spaces, Canadian Journal of Mathematics vol. 3 (1951) pp. 219-224.

5. L. Gillman, M. Henriksen, and M. Jerison, On a theorem of Gelfand and Kolmogoroff concerning maximal ideals in rings of continuous functions, Proc. Amer. Math. Soc. vol. 5 (1954) pp. 447-455.

6. F. Hausdorff, Grundzüge einer Theorie der geordneten Mengen, Math. Ann. vol. 65 (1908) pp. $435-505$.

7. - Grundzüge der Mengenlehre, Leipzig, 1914.

8. M. Henriksen, On the prime ideals of the ring of entire functions, Pacific Journal of Mathematics vol. 3 (1953) pp. 711-720.

9. M. Henriksen and J. R. Isbell, On the continuity of the real roots of an algebraic equation, Proc. Amer. Math. Soc. vol. 4 (1953) pp. 431-434.

10. E. Hewitt, Rings of real-valued continuous functions, I, Trans. Amer. Math. Soc. vol. 64 (1948) pp. 45-99.

11. - Linear functionals on spaces of continuous functions, Fund. Math. vol. 37 (1950) pp. 161-189.

12. I. Kaplansky, Topological rings, Amer. J. Math. vol. 69 (1947) pp. 153-183.

13. M. Katětov, Measures in fully normal spaces, Fund Math. vol. 38 (1951) pp. 73-84.

14. C. Kuratowski, Topologie I, Warsaw, 1933.

15. S. Lefschetz, Algebraic topology, Amer. Math. Soc. Colloquium Publications, vol. 27, 1942.

16. N. H. McCoy, Rings and ideals, Mathematical Association of America, 1948.

17. B. Pospísil, Remark on bicompact spaces, Ann. of Math. vol. 38 (1937) pp. 845-846.

18. T. Shirota, A class of topological spaces, Osaka Math. J. vol. 4 (1952) pp. 23-40.

19. R. Sikorski, Remarks on some topological spaces of high power, Fund. Math. vol. 37 (1950) pp. 125-136.

20. A. H. Stone, Paracompactness and product spaces, Bull. Amer. Math. Soc. vol. 54 (1948) pp. $977-982$.

21. M. H. Stone, Applications of the theory of Boolean rings to general topology, Trans. Amer. Math. Soc. vol. 41 (1937) pp. 375-481.

22. A. Tarski, Über unerreichbare Kardinalzahlen, Fund. Math. vol. 30 (1938) pp. 68-89.

23. — Drei Überdeckungssätze der allgemeinen Mengenlehre, Fund. Math. vol. 30 (1938) pp. 132-155.

24. A. Tychonoff, Über die topologische Erweiterung von Räumen, Math. Ann. vol. 102 (1930) pp. 544-561.

25. S. Ulam, Zur Masstheorie in der allgemeinen Mengenlehre, Fund. Math. vol. 16 (1930) pp. $140-150$.

26. B. L. van der Waerden, Moderne Algebra, Berlin, 1940.

PuRdue University, LAFAYETTE, IND. 